\title{
Spatial variations of PAH properties in M17SW revealed by Spitzer/IRS spectral mapping
}

\author{
M. Yamagishi ${ }^{1}$, H. Kaneda ${ }^{2}$, D. Ishihara ${ }^{2}$, S. Oyabu ${ }^{2}$, T. Suzuki ${ }^{2}$, T. Onaka ${ }^{3}$, T. Nagayama ${ }^{4}$, T.

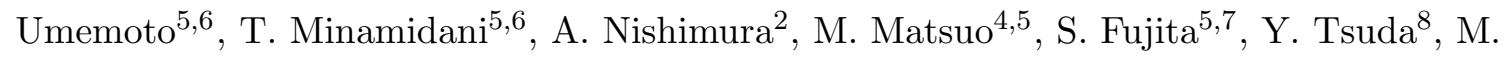 \\ $\mathrm{Kohno}^{2}$, and S. Ohashi ${ }^{3}$ \\ yamagish@ir.isas.jaxa.jp
}

\begin{abstract}
We present Spitzer/IRS mid-infrared spectral maps of the Galactic star-forming region M17 as well as IRSF/SIRIUS Br $\gamma$ and Nobeyama 45-m/FOREST ${ }^{13} \mathrm{CO}(J=1-$ $0)$ maps. The spectra show prominent features due to polycyclic aromatic hydrocarbons (PAHs) at wavelengths of $6.2,7.7,8.6,11.3,12.0,12.7,13.5$, and $14.2 \mu \mathrm{m}$. We find that the PAH emission features are bright in the region between the HII region traced by $\mathrm{Br} \gamma$ and the molecular cloud traced by ${ }^{13} \mathrm{CO}$, supporting that the $\mathrm{PAH}$ emission originates mostly from photo-dissociation regions. Based on the spatially-resolved Spitzer/IRS maps, we examine spatial variations of the PAH properties in detail. As a result, we find that the interband ratio of PAH $7.7 \mu \mathrm{m} / \mathrm{PAH} 11.3 \mu \mathrm{m}$ varies locally near M17SW, but rather independently of the distance from the OB stars in M17, suggesting that the degree of $\mathrm{PAH}$ ionization is mainly controlled by local conditions rather than the global UV environments determined by the OB stars in M17. We also find that the interband ratios of the PAH $12.0 \mu \mathrm{m}, 12.7 \mu \mathrm{m}, 13.5 \mu \mathrm{m}$, and $14.2 \mu \mathrm{m}$ features to the PAH $11.3 \mu \mathrm{m}$ feature are high near the M17 center, which suggests structural changes of PAHs through processing due to intense UV radiation, producing abundant edgy irregular PAHs near the M17 center.
\end{abstract}

\footnotetext{
${ }^{1}$ Institute of Space and Astronautical Science, Japan Aerospace Exploration Agency, Chuo-ku, Sagamihara 2525210, Japan

${ }^{2}$ Graduate School of Science, Nagoya University, Furo-cho, Chikusa-ku, Nagoya 464-8602, Japan

${ }^{3}$ Graduate School of Science, The University of Tokyo, 7-3-1 Hongo, Bunkyo-ku, Tokyo 113-0033, Japan

${ }^{4}$ Graduate School of Science and Engineering, Kagoshima University, 1-21-35 Korimoto, Kagoshima 890-0065, Japan

${ }^{5}$ Nobeyama Radio Observatory, National Astronomical Observatory of Japan (NAOJ), National Institutes of Natural Sciences (NINS), 462-2, Nobeyama, Minamimaki, Minamisaku, Nagano 384-1305, Japan

${ }^{6}$ Department of Astronomical Science, School of Physical Science, SOKENDAI (The Graduate University for Advanced Studies), 2-21-1, Osawa, Mitaka, Tokyo 181-8588, Japan

${ }^{7}$ Graduate School of Pure and Applied Sciences, University of Tsukuba, 1-1-1 Tennodai, Tsukuba, Ibaraki 3058577, Japan

${ }^{8}$ Graduate School of Science and Engineering, Meisei University, 2-1-1 Hodokubo, Hino, Tokyo 191-0042
} 
Subject headings: infrared: ISM, ISM: dust, photon-dominated region (PDR), individual object (M17 SW)

\section{Introduction}

Spectral bands due to polycyclic aromatic hydrocarbon (PAH) emissions are dominant features in the near- and mid-infrared (IR; 3-20 $\mu \mathrm{m})$. Since PAHs are excited by far-UV photons (6$13.6 \mathrm{eV}$ ), the PAH emission features are characteristic of photo-dissociation regions (PDRs). The PAH emission features are observed at wavelengths of 3.3, 6.2, 7.7, 8.6, 11.3, 12.0, 12.7, 13.5, 14.2, $15.8,16.4,17.4,17.8$ and $18.9 \mu \mathrm{m}$, which are attributed to vibrations of $\mathrm{C}-\mathrm{H}$ or $\mathrm{C}-\mathrm{C}$ bonds in hydrocarbons. Those features, especially main features at $6.2,7.7,8.6,11.3$, and $12.7 \mu \mathrm{m}$, are theoretically and observationally well-studied (e.g., Chan et al. 2001; Draine \& Li 2007; Tielens 2008; Bauschlicher et al. 2009). Past studies have shown that the PAH interband ratios are useful probes to study the properties of PAHs. Among these, the degree of PAH ionization is best studied through interband ratios involving the $6.2,7.7$ and $11.3 \mu \mathrm{m}$ PAH interband ratios. Here the 6.2 and $7.7 \mu \mathrm{m}$ bands, due to C-C vibrations, are representative of ionized PAHs and the $11.3 \mu \mathrm{m}$ band, due to C-H vibrations, representative of neutral PAHs (e.g., Allamandola et al. 1999; Peeters et al. 2002). It is expected that the degree of PAH ionization is relatively high near exciting sources while that is low in molecular clouds. Another important property is the edge structure of PAHs, which is probed by using the interband ratios of the PAH features at 11.3, 12.0, 12.7, 13.5, and $14.2 \mu \mathrm{m}$; likely origins of these features are all $\mathrm{C}-\mathrm{H}$ in-plane bending, but the numbers of adjacent C-H bonds in a benzene ring are different (PAH $11.3 \mu \mathrm{m}$ : solo; PAH $12.0 \mu \mathrm{m}$ : duo; PAH $12.7 \mu \mathrm{m}$ : trio; PAH $13.5 \mu \mathrm{m}$ and PAH $14.2 \mu \mathrm{m}$ : quartet; Draine 2003; Tielens 2005). For example, edgy PAHs are expected to show the strong PAH 12.0, 12.7, 13.5, and $14.2 \mu \mathrm{m}$ features relative to the $\mathrm{PAH} 11.3 \mu \mathrm{m}$ feature. The structures of PAHs may change from region to region depending on the surrounding radiation field (Boersma et al. 2013; Kaneda et al. 2014). Therefore examining the PAH ionization together with the PAH edge structure may be helpful to discuss variations of the PAH properties.

Recently, variations in the PAH properties have been intensively studied for a variety of targets mainly with Spitzer and AKARI (e.g., Peeters et al. 2002; Smith et al. 2007b; Boersma et al. 2012; Yamagishi et al. 2012). Most of such studies, however, discussed PAHs in individual areas, and did not intensively examine spatial variations of PAHs. In order to examine the effects of the surrounding interstellar environment on the PAH properties, spatially-resolved observations are essential (e.g. Crété et al. 1999; Rapacioli et al. 2005; Berné et al. 2007; Sakon et al. 2007; Kaneda et al. 2005, 2008; Fleming et al. 2010; Yamagishi et al. 2010; Berné \& Tielens 2012; Pilleri et al. 2012; Egusa et al. 2013; Croiset et al. 2016). One of the most intensive spatially-resolved studies in PAHs was carried out by Boersma et al. (2013, 2014, 2015). They analyzed Spitzer/IRS spectral maps of the reflection nebula NGC 7023 and decomposed the observed spectra to the emission fea-

tures from ionized and neutral PAHs. As a result, they found clear spatial variations in the degree 
of PAH ionization along the direction from the exciting B-type star to PDR and molecular-cloud regions. Stock et al. (2016) also examined the PAH interband ratios of seven Galactic HII regions and three reflection nebulae with Spitzer/IRS spectral maps, although they did not discuss spatial variations of the ratios in the target regions. Haraguchi et al. (2012) examined the degree of PAH ionization in the Orion nebula based on their ground-based near-IR narrow-band observations. The number of such spatially-resolved studies of PAHs is, however, still limited.

In this paper, we present Spitzer/IRS spectral maps of the mid-IR PAH features in the Galactic star-forming region M17 as well as $\mathrm{Br} \gamma$ and ${ }^{13} \mathrm{CO}(J=1-0)$ maps covering the same region. M17 is young ( 1 Myr; Hanson et al. 1997) and one of the well-studied active Galactic star-forming regions (e.g., Stutzki \& Guesten 1990; Giard et al. 1992, 1994; Cesarsky et al. 1996; Povich et al. 2007), which contains more than 100 OB stars in the central cluster, NGC 6618 (Lada et al. 1991). Among them, the most active ionizing source is CEN1, a binary of O4+O4 stars (Chini et al. 1980). Assuming the distance of $2 \mathrm{kpc}$ ( $\mathrm{Xu}$ et al. 2011), the area of the spectral maps in the present study is $1.2 \times 1.2 \mathrm{pc}^{2}$ which is 80 times larger than that in the study of NGC 7023 (Boersma et al. 2013). Based on the wide-area spectral maps, we examine the effects of the intense star-forming activity on the properties of PAHs in detail.

\section{Observations and Data Reduction}

\subsection{Spitzer/IRS}

We analyzed archival Spitzer/IRS spectral mapping data (AORKEY: 17976320, 17976576, 17977344, and 17977600), which were retrieved from the Spitzer Heritage Archives. The observed area is shown in Fig. 1. The used module is short-wavelength-low-resolution (SL) covering a wavelength range of 5.2-14.5 $\mu \mathrm{m}$ with a spectral resolution of $R=64-128$ (Houck et al. 2004). We used CUbe Builder for IRS Spectra Maps ver. 1.8 (CUBISM; Smith et al. 2007a) with the default setting to reduce the data, and obtained spectral and its uncertainty cubes for the three orders named SL1 $(7.4-14.5 \mu \mathrm{m})$, SL2 $(5.2-7.7 \mu \mathrm{m})$, and SL3 $(7.3-8.6 \mu \mathrm{m})$, separately. We checked bad pixels with eyes and removed them. In the data reduction, background spectra were not considered. The major background in the mid-IR is the zodiacal light, the spectra of which have smooth continua without prominent spectral features (Reach et al. 1996). The intensity of the zodiacal light is $\sim 60 \mathrm{MJy} / \mathrm{sr}$ at a wavelength of $18 \mu \mathrm{m}$ around M17 (Kondo et al. 2016). The contribution of the zodiacal light in M17 spectra is negligible near the center, while that is not in outer regions. We, therefore, do not discuss the continuum emission in the present study. After unifying the pixel scale from the original size to $3 .^{\prime \prime} 6$ for the three FITS cubes to correct differences in the spatial resolution at each wavelength range, the intensity levels for SL1 and SL2 were adjusted to coincide with SL3 in the overlapped wavelength ranges. Finally, we obtained 990 independent mid-IR spectra. 


\subsection{IRSF/SIRIUS}

We performed narrow-band imaging of M17 with the SIRIUS camera on the IRSF $1.4 \mathrm{~m}$ telescope (Nagashima et al. 1999; Nagayama et al. 2003). SIRIUS has a field of view of $7 .^{\prime} 7 \times$ 7.'7 with a pixel scale of $0 .{ }^{\prime \prime} 45$. We observed M17 using the two narrow-band filters tuned for the $\mathrm{Pa} \beta 1.28 \mu \mathrm{m}$ and $\operatorname{Br} \gamma 2.16 \mu \mathrm{m}$ lines, simultaneously. The effective band widths of the filters are $0.029 \mu \mathrm{m}$ for $\mathrm{Pa} \beta$ and $0.038 \mu \mathrm{m}$ for $\mathrm{Br} \gamma$. The observation was carried out on 2013 June 10 with an integration time of 75 seconds and 20 dithering. The observed area is also shown in Fig. 1, In the present study, we analyzed only the $\operatorname{Br} \gamma$ image which is less affected by the interstellar extinction than the $\mathrm{Pa} \beta$ image.

We reduced the image data based on the standard data reduction procedure including dark subtraction, flat-fielding, sky subtraction and dithered-image-combining. Since we did not subtract a continuum image, free-free continuum emission may be contaminated in the $\mathrm{Br} \gamma$ image. Photometric calibration was performed by comparing point-source fluxes in the image with those in the 2MASS Point Source Catalog (Skrutskie et al. 2006), where we assumed that the magnitude of each point source is the same between the $K_{s}$-band and the narrow-band images. We used sufficiently isolated (11) point sources with the $K_{s}$-band flux in a range of 9.0-11.0 mag and with errors smaller than 0.05 mag. As a result, the uncertainty of the photometric calibration coefficient is $\sim 5 \%$. The final $1 \sigma$ noise level is $7.0 \times 10^{-9} \mathrm{~W} / \mathrm{m}^{2} / \mathrm{sr}$.

\subsection{Nobeyama 45-m/FOREST}

We analyzed CO mapping data of M17, which were taken in the framework of the FUGIN (FOREST Ultra-Wide Galactic plane survey In Nobeyama; Minamidani et al. 2016b) legacy survey covering a wide area of the Galactic plane $\left(l=10-50^{\circ}, 198-236^{\circ},|b|<1^{\circ}\right)$ with a high spatial resolution of $15^{\prime \prime}$ and $1 \sigma$ noise level of $0.4 \mathrm{~K}$ in the $T_{\mathrm{mb}}$ scale at $1 \mathrm{~km} / \mathrm{s}$ velocity resolution. FOREST (Minamidani et al. 2016a) is a four-beam receiver, and has a capability of simultaneous on-the-fly mapping observations using the three $\mathrm{CO}$ lines, ${ }^{12} \mathrm{CO}(J=1-0),{ }^{13} \mathrm{CO}(J=1-0)$, and $\mathrm{C}^{18} \mathrm{O}(J=1-0)$. Pointing errors were corrected every 1.5 hours by observing $\mathrm{SiO}$ maser sources. As a result, the pointing accuracy of the telescope is kept to be $<5^{\prime \prime}$. We used calibrated ${ }^{13} \mathrm{CO}$ map (internal team release version 1.3) because it is likely optically thin and traces overall molecular cloud structures in star forming regions. The ${ }^{13} \mathrm{CO}$ map has spatial resolution of $18^{\prime \prime}$ and $1 \sigma$ noise level of $\sim 0.8 \mathrm{~K}$ in

the $T_{\mathrm{mb}}$ scale at $1 \mathrm{~km} / \mathrm{s}$ velocity resolution as an intermediate product of the survey project. The detail of the survey observations and data reductions will be described in Umemoto et al. in prep. 


\section{Analyses and Results}

Figure 2 shows examples of the spectra extracted from positions I, II, and III which are labeled in Fig. 1. The spectra show a variety of emission features due to PAHs at 6.2, 7.7, 8.6, 11.3, 12.0, $12.7,13.5$, and $14.2 \mu \mathrm{m}$, and fine-structure lines ([NeII], [ArIII], and [SIV]). The fine-structure lines are dominant at position I which is nearest to the M17 center, while the PAH features are dominant at positions II and III. In order to examine variations of the PAH intensities and interband ratios, we decomposed the spectra using PAHFIT (Smith et al. 2007b), which is designed to fit various spectral features in Spitzer/IRS SL spectra including silicate dust absorption around $9.7 \mu \mathrm{m}$. We used PAHFIT assuming screen dust extinction, and reconstructed the spectral maps of the mid-IR features. In the mid-IR spectra, the PAH $7.7 \mu \mathrm{m}, 11.3 \mu \mathrm{m}$, and $12.7 \mu \mathrm{m}$ features are treated as PAH complexes which have multiple components. As treated in Smith et al. (2007b), we calculated a sum of the three components centered at $7.42 \mu \mathrm{m}, 7.60 \mu \mathrm{m}$, and $7.85 \mu \mathrm{m}$ for the intensity of the PAH $7.7 \mu \mathrm{m}$ complex, the two components centered at $11.23 \mu \mathrm{m}$ and $11.33 \mu \mathrm{m}$ for the intensity of the PAH $11.3 \mu \mathrm{m}$ complex, and the two components centered at $12.62 \mu \mathrm{m}$ and $12.69 \mu \mathrm{m}$ for the intensity of the PAH $12.7 \mu \mathrm{m}$ complex. In the fitting procedure, we considered the systematic error of $2 \%$ (Lebouteiller et al. 2011) in addition to random errors provided by CUBISM. Figure 2 also shows the results of PAHFIT, where PAHFIT reproduces the example spectra very well.

Figure 3 shows the resultant spectral maps obtained for the mid-IR features (PAH features at $6.2,7.7,8.6,11.3,12.0,12.7,13.5$, and $14.2 \mu \mathrm{m}$, [NeII], [ArIII], [SIV], and optical depth of silicate dust absorption at $9.7 \mu \mathrm{m}$ ), where the interstellar extinction is corrected. In the maps, there is a large-scale gradient from the north-east to the south-west. In the PAH maps, there are also local structures especially on the east side, suggesting that PAHs are not only globally but also locally affected by the surrounding interstellar environments. It is notable that the maps of the PAH $12.7 \mu \mathrm{m}$ and $12.0 \mu \mathrm{m}$ features are similar to each other. They are both probably attributed to C-H out-of-plane bending modes, and the former is blended with the strong [NeII] line, while the latter is not. Therefore, this similarity suggests that PAHFIT has successfully decomposed emissions from the PAH $12.7 \mu \mathrm{m}$ feature and the [NeII] line. In the fine-structure line maps, a spatial extent in the [NeII] map is large, while that in [SIV] is small, which presumably corresponds to the difference in the ionization potential; the energies required to ionize $\mathrm{Ne}^{0}, \mathrm{Ar}^{+}$, and $\mathrm{S}^{++}$are $21.6 \mathrm{eV}, 27.6 \mathrm{eV}$, and $34.8 \mathrm{eV}$, respectively. In the optical depth map, there is a ridge structure in the north-south direction. We confirm that the optical depth estimated by using PAHFIT is consistent with that estimated in Stock et al. (2016).

Figure 4 shows the PAH interband ratios of the PAH $6.2 \mu \mathrm{m}, 8.6 \mu \mathrm{m}, 11.3 \mu \mathrm{m}$, and $12.7 \mu \mathrm{m}$ features to the PAH $7.7 \mu \mathrm{m}$ feature plotted against intensities of the PAH $7.7 \mu \mathrm{m}$ feature. The dashed lines in Fig. 4 indicate the best-fit power-law relation for each of the PAH interband ratio. It is notable that the best-fit lines are nearly flat, indicating that there are no clear systematic variations in the PAH interband ratios for various interstellar environments. By contrast, there are clear differences in the dispersion along the vertical axis from ratio to ratio. We calculated $1 \sigma$ standard deviations of the PAH interband ratios. As a result, we find that the PAH $6.2 \mu \mathrm{m} / \mathrm{PAH} 7.7 \mu \mathrm{m}$ 
ratios show the smallest variations (PAH $6.2 \mu \mathrm{m} / \mathrm{PAH} 7.7 \mu \mathrm{m}$ : 0.028 dex (decimal exponent), PAH $8.6 \mu \mathrm{m} / \mathrm{PAH} 7.7 \mu \mathrm{m}$ : 0.065 dex, PAH $11.3 \mu \mathrm{m} / \mathrm{PAH} 7.7 \mu \mathrm{m}: 0.086 \mathrm{dex}$, PAH $12.7 \mu \mathrm{m} / \mathrm{PAH} 7.7 \mu \mathrm{m}:$ 0.11 dex). Probable origins of the PAH $6.2 \mu \mathrm{m}$ and $7.7 \mu \mathrm{m}$ features are C-C bonds, while those of the other PAH features are C-H bonds (Allamandola et al. 1989; Draine \& Li 2007). Therefore, the difference in the variations in the interband ratios may reflect spatial variations in the properties of the PAH population.

Figure 5(a) shows the ${ }^{13} \mathrm{CO}(J=1-0)$ contour map overlaid on the $\operatorname{Br} \gamma$ image of M17. The $\operatorname{Br} \gamma$ emission is detected in an inner region of M17, especially in south-west and north-east areas, but not detected in west and south-west areas. In the regions where $\mathrm{Br} \gamma$ is not detected, the ${ }^{13} \mathrm{CO}$ emission is strongly detected. The strong peak of the ${ }^{13} \mathrm{CO}$ emission is adjacent to the bright rim of the $\mathrm{Br} \gamma$ emission. Thanks to the high spatial resolution of the ${ }^{13} \mathrm{CO}$ map and the small extinction in the $\mathrm{Br} \gamma$ map, a spatial separation of an HII region from the molecular cloud is clearly recognized. Figure 5(b) shows comparison of the total major PAH map (PAH $6.2 \mu \mathrm{m}+\mathrm{PAH} 7.7 \mu \mathrm{m}+\mathrm{PAH}$ $8.6 \mu \mathrm{m}+\mathrm{PAH} 11.3 \mu \mathrm{m}+\mathrm{PAH} 12.7 \mu \mathrm{m}$ ) with the ${ }^{13} \mathrm{CO}$ map. By comparing Fig. 5 (a) with Fig. 5(b), it is clear that the $\mathrm{PAH}$ emission is bright on the boundary between the $\mathrm{Br} \gamma$ and ${ }^{13} \mathrm{CO}$ emissions, which strongly supports that the PAH features are emitted from PDRs.

Figure 6 shows the PAH interband ratio maps (PAH $6.2 \mu \mathrm{m} / \mathrm{PAH} 11.3 \mu \mathrm{m}, \mathrm{PAH} 7.7 \mu \mathrm{m} / \mathrm{PAH}$ $11.3 \mu \mathrm{m}$, and $\mathrm{PAH} 8.6 \mu \mathrm{m} / \mathrm{PAH} 11.3 \mu \mathrm{m}$ ) and their correlation plots. The interband ratios are possible probes of the degree of PAH ionization because the PAH 6.2, 7.7, and $8.6 \mu \mathrm{m}$ features are efficiently emitted by ionized PAHs, while the PAH $11.3 \mu \mathrm{m}$ feature is emitted by both ionized and neutral PAHs (Allamandola et al. 1999; Peeters et al. 2002). In the maps, there is no clear large-scale gradient as seen in Fig. 3, and local structures are dominant, suggesting that the PAH properties are controlled by local conditions rather than the large-scale UV environment determined by the OB stars in M17. It is notable that these ratios are high near the ${ }^{13} \mathrm{CO}$ peak. Position II in Fig. 1 corresponds to the peak of the PAH $7.7 \mu \mathrm{m} / \mathrm{PAH} 11.3 \mu \mathrm{m}$ ratio. The spectrum extracted from position II actually shows a high PAH $7.7 \mu \mathrm{m} / \mathrm{PAH} 11.3 \mu \mathrm{m}$ ratio in comparison with those extracted from positions I and III (Fig. 2). This result supports that the degree of PAH ionization is significantly different from position to position. The PAH $6.2 \mu \mathrm{m} / \mathrm{PAH} 11.3 \mu \mathrm{m}$ ratios tightly correlate with the PAH $7.7 \mu \mathrm{m} / \mathrm{PAH} 11.3 \mu \mathrm{m}$ ratios, while they do not tightly correlate with the PAH $8.6 \mu \mathrm{m} / \mathrm{PAH} 11.3 \mu \mathrm{m}$ ratios, although the PAH $8.6 \mu \mathrm{m} / \mathrm{PAH} 11.3 \mu \mathrm{m}$ ratio is also known to be a measure of the PAH ionization (e.g., Boersma et al. 2014). Since the probable origin of the PAH $8.6 \mu \mathrm{m}$ feature (C-H in-plane-bending mode; Tielens 2005) is different from that of the PAH $6.2 \mu \mathrm{m}$ and $7.7 \mu \mathrm{m}$ features (C-C stretching mode; Tielens 2005), other factors (e.g., size) may affect the PAH $8.6 \mu \mathrm{m} / \mathrm{PAH} 11.3 \mu \mathrm{m}$ ratio (e.g., Bauschlicher et al. 2008, 2009; Ricca et al. 2012). Maps and correlation plots of other four interband ratios (PAH $12.0 \mu \mathrm{m} / \mathrm{PAH} 11.3 \mu \mathrm{m}$, PAH $12.7 \mu \mathrm{m} / \mathrm{PAH} 11.3 \mu \mathrm{m}$, PAH $13.5 \mu \mathrm{m} / \mathrm{PAH} 11.3 \mu \mathrm{m}$, and $\mathrm{PAH} 14.2 \mu \mathrm{m} / \mathrm{PAH} 11.3 \mu \mathrm{m})$ are shown in Fig. 7, which are likely probes of the PAH edge structure. The maps show that these ratios are relatively high on the near side of M17, suggesting that PAH structures are not uniform in the region. The plots show global correlations among these interband ratios, supporting the 
same origin for these features (i.e., C-H out-of-plane bending mode).

In order to check robustness of the above results, we also evaluated the PAH features in the following model-independent manner: we used linear baselines of 5.8-6.5 $\mu \mathrm{m}, 7.1-8.3 \mu \mathrm{m}, 8.3^{-}$ $8.8 \mu \mathrm{m}, 10.8-11.8 \mu \mathrm{m}$, and 13.8-14.5 $\mu \mathrm{m}$ for the PAH $6.2 \mu \mathrm{m}, 7.7 \mu \mathrm{m}, 8.6 \mu \mathrm{m}, 11.3 \mu \mathrm{m}$, and $14.2 \mu \mathrm{m}$ features, respectively, to estimate their intensities. Figures 8, 9(a)-9(c), and 9(d) correspond to Figs. 3. 6(a)-6(c), and 7(d), as obtained in this method. We confirm from these figures that the linear-baseline-fitting results show overall consistency with the PAHFIT result. Galliano et al. (2008) showed that the PAH interband ratios are mostly independent of the approach isolating the PAH features.

\section{Discussion}

\subsection{Spatial variations in the degree of $\mathrm{PAH}$ ionization}

We find that the PAH $7.7 \mu \mathrm{m} / \mathrm{PAH} 11.3 \mu \mathrm{m}$ ratio ranges from 2.6 to 9.0 with a median value of 5.2, suggesting that the degree of PAH ionization is significantly variable in M17SW. In order to derive a map of the degree of PAH ionization, we estimate the degree of ionization from the PAH $7.7 \mu \mathrm{m} / \mathrm{PAH} 11.3 \mu \mathrm{m}$ ratio using the following equation in Joblin et al. (1996):

$$
\frac{I_{7.7}}{I_{11.3}}=\frac{I_{7.7}^{0}}{I^{0}{ }_{11.3}} \frac{\left(1+\frac{n^{+}}{n^{0}} \frac{I^{+} 7.7}{I^{0} 7.7}\right)}{\left(1+\frac{n^{+}}{n^{0}} \frac{I^{+} 11.3}{I^{0} 11.3}\right)}
$$

where $I_{7.7}, I_{11.3}$, and $n$ indicate the intensities of the PAH $7.7 \mu \mathrm{m}$ and PAH $11.3 \mu \mathrm{m}$ features, and the number density of PAHs, respectively. The zero and plus in the superscript indicate the neutral and ionized states, respectively. We assumed $I^{0}{ }_{7.7} / I^{0}{ }_{11.3}, I^{+}{ }_{7.7} / I^{0}{ }_{7.7}$, and $I^{+}{ }_{11.3} / I^{0}{ }_{11.3}$ to be 1.3 , 5.54, and 0.6, respectively (Fleming et al. 2010), and converted the observed $I_{7.7} / I_{11.3}$ to $n^{+} / n^{0}$. Finally, we obtained the degree of PAH ionization of $\left(1+n^{0} / n^{+}\right)^{-1}$.

Figure 10 shows a map of the degree of $\mathrm{PAH}$ ionization thus derived. In the figure, the degree of PAH ionization ranges from $19 \%$ to $81 \%$ with a median value of $48 \%$. These values correspond to the ionization parameter, $G_{0} \sqrt{T_{\text {gas }}} / n_{e}$, of $9 \times 10^{3}$ to $1 \times 10^{5}$ (Tielens 2005), where $G_{0}$, $T_{\text {gas }}$, and $n_{e}$ indicate the far-UV intensity normalized by that of the solar neighborhood, gas temperature, and electron density, respectively, and we assumed the number of carbon atoms in a PAH to be 50 . Therefore, the degree of PAH ionization varies in a wide range from region to region, and typical PAHs are moderately ionized in M17SW. Such a situation is similar to the cases of the previous studies; the degree of PAH ionization is 10-70\% and typically $50 \%$ in NGC 7023 (Boersma et al. 2013), while that is 20-100 \% and typically $50 \%$ in the Orion Bar (Haraguchi et al. 2012), although the approaches to determine the degree of $\mathrm{PAH}$ ionization are different between those studies and the present one. The minimum degree of PAH ionization of $\sim 15 \%$ is common among the three

objects, suggesting that PAHs in molecular clouds adjacent to star-forming regions are slightly 
ionized. The maximum degree of $\mathrm{PAH}$ ionization of $80 \%$ in $\mathrm{M} 17 \mathrm{SW}$ is lower than that of Orion Bar and comparable to that in NGC 7023. This difference is presumably due to the difference in the observed positions; the exciting stars are covered in the observational area of Orion Bar, while those are not covered in those of M17 and NGC 7023.

One interesting result in Fig. 10 is that the degree of PAH ionization is high near the peak of the ${ }^{13} \mathrm{CO}$ emission. As expressed in the definition of the ionization parameter, the balance between the ionization and recombination is important in the degree of PAH ionization. The ionization depends on the far-UV radiation field, $G_{0}$, while the recombination mainly depends on the electron density, $n_{e}$. Therefore, we evaluate $G_{0}$ and $n_{e}$ to verify the spatial variation of the degree of PAH ionization. We determined $G_{0}$ with the Herschel/PACS 70 and $100 \mu \mathrm{m}$ maps which we retrieved from NASA/IPAC Infrared Science Archives (Observation ID: 1342192767). We converted a $70 \mu \mathrm{m} / 100 \mu \mathrm{m}$ color temperature to $G_{0}$ assuming the power-law dust emissivity index of $\beta=1$ and the relation between $G_{0}$ and $T_{\text {dust }}, G_{0}=\left(T_{\text {dust }} / 17.5\right)^{5}$ (Boulanger et al. 1996). The derived $G_{0}$ map is shown in Fig. 111, where obvious HII regions show strong [NeII] $\left(>5.0 \times 10^{-6} \mathrm{~W} / \mathrm{m}^{2} / \mathrm{sr}\right)$ are masked out as the conversion is not applicable to HII regions. Electrons in PDRs are mainly produced by ionization of neutral carbon atoms. Therefore, $[\mathrm{CII}]$ is a possible probe of $n_{e}$ for a nearly constant gas temperature; a [CII] map of this region is obtained by Pérez-Beaupuits et al. (2012).

In Fig. 11, we find that the $G_{0}$ map exhibits a local maximum near the peak of the degree of PAH ionization, whereas the [CII] map in Pérez-Beaupuits et al. (2012) does not have a local minimum there. These results support the high degree of PAH ionization inside the molecular cloud, which is driven by high $G_{0}$ rather than low $n_{e}$. Although M17SW is one of the most intensively studied regions in our Galaxy, the spatial structure in the line of sight is still unclear; Stutzki \& Guesten (1990) and Sheffer \& Wolfire (2013) proposed clumpy and bowl-shaped structures in M17SW, respectively. Therefore the local peak of the degree of PAH ionization may be due to geometrical effects, and in reality, this region can be located closely to the M17 center. This situation is, however, rather unlikely, because Fig. 7 shows no corresponding local peak in the interband ratios; such a region should also show variations in the PAH structure due to the intense UV radiation from the M17 center. It should be noted that the region with high PAH ionization degree is associated with dense gas probed by ${ }^{13} \mathrm{CO}$. Additionally, since PAHs are ionized by farUV photons, B-type or later stars can substantially ionize PAHs. In this situation, such stars may be reasonable ionizing source candidates because the $\operatorname{Br} \gamma$ emission is not locally enhanced in the region (Fig. 5). Therefore, it is possible that B-type or later stars buried inside the molecular cloud locally ionize PAHs. As can be seen in Fig. 11, an even stronger $G_{0}$ peak is found at $\sim 30^{\prime \prime}$ to the south, where the degree of PAH ionization does not show a significant increase (Fig. 10). Young stellar objects which cannot substantially ionize PAHs may attribute to the $G_{0}$ peak here. 


\subsection{Spatial variations of the PAH structure}

In Figs. 7(a)-7(d), the ratios of the PAH 12.0, 12.7, 13.5, and $14.2 \mu \mathrm{m}$ features to the PAH $11.3 \mu \mathrm{m}$ feature have a strong peak near the M17 center. These interband ratios are possible probes of the PAH edge structures. Likely origins of these features are all $\mathrm{C}-\mathrm{H}$ out-of-plane bending, but the numbers of adjacent $\mathrm{C}-\mathrm{H}$ bonds in a benzene ring are different (PAH $11.3 \mu \mathrm{m}$ : solo; PAH $12.0 \mu \mathrm{m}$ : duo; PAH $12.7 \mu \mathrm{m}$ : trio; PAH $13.5 \mu \mathrm{m}$ and PAH $14.2 \mu \mathrm{m}$ : quartet; Honv et al. 2001; Draine 2003; Tielens 2005). Therefore, Fig. 7 suggests that the PAH structure is not uniform in the observed region, but is different especially near the M17 center.

Among the interband ratios, the PAH $13.5 \mu \mathrm{m} / \mathrm{PAH} 11.3 \mu \mathrm{m}$ and the PAH $14.2 \mu \mathrm{m} / \mathrm{PAH}$ $11.3 \mu \mathrm{m}$ ratios are more localized toward the center of M17 relative to the PAH $12.0 \mu \mathrm{m} / \mathrm{PAH}$ $11.3 \mu \mathrm{m}$ and the PAH $12.7 \mu \mathrm{m} / \mathrm{PAH} 11.3 \mu \mathrm{m}$ ratios. Pencil-beam observations by Hony et al. (2001) observationally showed that the PAH $13.5 \mu \mathrm{m} / \mathrm{PAH} 11.3 \mu \mathrm{m}$ and the PAH $14.2 \mu \mathrm{m} / \mathrm{PAH}$ $11.3 \mu \mathrm{m}$ ratios in their HII-region samples tend to be higher than those in PDR samples. Our spectral maps clearly show such spectral variations in M17. One possible interpretation of the high interband ratios is processing of edgy PAHs. Since the survival time of edgy PAHs is short, edgy PAHs observed near the M17 center may be freshly released ones from grain mantles due to the intense UV radiation. Hence Figs. [7(a)-7(d) may suggest the active PAH erosion/destruction process at the PDR surface.

The plots in Figs. $7(\mathrm{e})-7(\mathrm{~h})$ show overall good correlations between the interband ratios. Boersma et al. (2015) showed that the PAH $12.7 \mu \mathrm{m} / \mathrm{PAH} 11.3 \mu \mathrm{m}$ ratio depends on both the degree of PAH ionization and the PAH edge structure. Since the spatial positions showing structural changes and high degree of ionization are different in the present study, our data may be useful to examine the dependence of the edge structure and the degree of PAH ionization on the PAH $12.7 \mu \mathrm{m} / \mathrm{PAH} 11.3 \mu \mathrm{m}$ ratio. In Figs. 7(e)-7(g), the PAH $12.7 \mu \mathrm{m} / \mathrm{PAH} 11.3 \mu \mathrm{m}$ ratios show good correlation with the ratios probing the PAH edge structure; the correlation coefficients are $R=0.78(N=399), 0.76(N=284), 0.92(N=111)$, respectively. In Fig. 12, we also examine the correlation between the PAH $12.7 \mu \mathrm{m} / \mathrm{PAH} 11.3 \mu \mathrm{m}$ ratios with the PAH $7.7 \mu \mathrm{m} / \mathrm{PAH} 11.3 \mu \mathrm{m}$ ratios probing the degree of $\mathrm{PAH}$ ionization. In the figure, correlation is relatively weak $(R=0.28$, $N=418$ ), suggesting that the PAH $12.7 \mu \mathrm{m} / \mathrm{PAH} 11.3 \mu \mathrm{m}$ ratios depend more strongly on the PAH edge structure rather than the degree of PAH ionization in M17.

Comparing Figs. $7(\mathrm{e})-\mathbf{7}(\mathrm{h})$ in detail, we find that there are small differences in the relation; the PAH $12.0 \mu \mathrm{m} / \mathrm{PAH} 11.3 \mu \mathrm{m}$ and PAH $13.5 \mu \mathrm{m} / \mathrm{PAH} 11.3 \mu \mathrm{m}$ ratios increase nearly in proportion to the PAH $12.7 \mu \mathrm{m} / \mathrm{PAH} 11.3 \mu \mathrm{m}$ and PAH $14.2 \mu \mathrm{m} / \mathrm{PAH} 11.3 \mu \mathrm{m}$ ratios, respectively (Figs. Z(e) and $7(\mathrm{~h})$ ). On the other hand, the PAH $13.5 \mu \mathrm{m} / \mathrm{PAH} 11.3 \mu \mathrm{m}$ and PAH $14.2 \mu \mathrm{m} / \mathrm{PAH} 11.3 \mu \mathrm{m}$ ratios do not increase in proportion to the $\mathrm{PAH} 12.7 \mu \mathrm{m} / \mathrm{PAH} 11.3 \mu \mathrm{m}$ ratio, and there are apparent

offsets in their relations (Figs. Z(f) and 7(g)). Hence, the PAH $12.0 \mu \mathrm{m}$ and $12.7 \mu \mathrm{m}$ intensities start to increase first and then increases in the PAH $13.5 \mu \mathrm{m}$ and $14.2 \mu \mathrm{m}$ intensities follow, relative to the PAH $11.3 \mu \mathrm{m}$ intensity. The relative delay in the growth of the PAH $13.5 \mu \mathrm{m}$ 
and $14.2 \mu \mathrm{m}$ intensities suggests that processing of PAHs may gradually take place due to the intense (and/or hard) UV. The proportionality of the PAH $13.5 \mu \mathrm{m}$ intensity to the PAH $14.2 \mu \mathrm{m}$ intensity (Fig. $7(\mathrm{~h})$ ) supports that they are of the same origin (quartet C-H in-plane bending mode), as suggested by Tielens (2005).

\section{Conclusion}

Based on Spitzer/IRS spectral mapping observations, we have examined spatial variations of the PAH properties around the M17SW region. We analyzed independent 990 spectra, which show prominent PAH features at wavelengths of $6.2,7.7,8.6,11.3,12.0,12.7,13.5$, and $14.2 \mu \mathrm{m}$ as well as fine-structure lines. We decomposed all the spectral features using PAHFIT (Smith et al. 2007b). As a result, the derived PAH emissions are bright in regions between HII regions traced by $\mathrm{Br} \gamma$ and molecular cloud regions traced by ${ }^{13} \mathrm{CO}$. Additionally, the PAH intensity maps show a large-scale gradient from the north-east to south-west, indicating that PAHs are irradiated by UV from the OB stars in the M17 center. By contrast, PAH interband ratio maps show no clear large-scale gradient but they are locally changed. These results suggest that the PAH ionization is mainly controlled by local conditions rather than the large-scale UV environment determined by the OB stars in the M17 center. The degree of PAH ionization estimated from the PAH $7.7 \mu \mathrm{m} / \mathrm{PAH} 11.3 \mu \mathrm{m}$ ratios ranges from 19 to $81 \%$ with a median value of $48 \%$, which is comparable to that in the previous studies for NGC 7023 and the Orion Bar. We also find that the degree of PAH ionization is high near the peak of the ${ }^{13} \mathrm{CO}$ emission. We discuss the ionization balance using the $G_{0}$ and [CII] maps to find that $G_{0} /[\mathrm{CII}]$ ratios show a local maximum inside the molecular cloud. We conclude that buried B-type or later stars may be important to determine the degree of PAH ionization in local conditions. Additionally, the PAH edge structures are examined by ratios of the PAH 12.0, 12.7, 13.5, and $14.2 \mu \mathrm{m}$ features to the PAH $11.3 \mu \mathrm{m}$ feature, which suggests that edgy PAHs are processed due to the intense (and/or hard) UV radiation especially near the M17 center.

This work is based on archival data obtained with the Spitzer Space Telescope, which is operated by the Jet Propulsion Laboratory, California Institute of Technology under a contract with NASA, and with the Herschel Space Observatory. This work is also based on observations with IRSF and the 45-m telescope in the Nobeyama Radio Observatory (NRO). The operation of IRSF is supported by Joint Development Research of National Astronomical Observatory of Japan, and Optical Near-Infrared Astronomy Inter-University Cooperation Program, funded by the the Ministry of Education, Culture, Sports, Science and Technology of Japan. NRO is a branch of the National Astronomical Observatory of Japan, National Institutes of Natural Sciences. This research was supported by JSPS KAKENHI Grant Number 25247020. S. Ohashi is financially

supported by a Research Fellowship from JSPS for Young Scientists. We also express many thanks to the anonymous referee for the useful comments. 


\section{REFERENCES}

Allamandola, L. J., Hudgins, D. M., \& Sandford, S. A. 1999, ApJ, 511, L115

Allamandola, L. J., Tielens, A. G. G. M., \& Barker, J. R. 1989, ApJS, 71, 733

Bauschlicher, Jr., C. W., Peeters, E., \& Allamandola, L. J. 2008, ApJ, 678, 316

—. 2009, ApJ, 697, 311

Berné, O., \& Tielens, A. G. G. M. 2012, Proceedings of the National Academy of Science, 109, 401

Berné, O., Joblin, C., Deville, Y., et al. 2007, A\&A, 469, 575

Boersma, C., Bregman, J., \& Allamandola, L. J. 2014, ApJ, 795, 110

—. 2015, ApJ, 806, 121

Boersma, C., Bregman, J. D., \& Allamandola, L. J. 2013, ApJ, 769, 117

Boersma, C., Rubin, R. H., \& Allamandola, L. J. 2012, ApJ, 753, 168

Boulanger, F., Abergel, A., Bernard, J.-P., et al. 1996, A\&A, 312, 256

Cesarsky, D., Lequeux, J., Abergel, A., et al. 1996, A\&A, 315, L309

Chan, K.-W., Roellig, T. L., Onaka, T., et al. 2001, ApJ, 546, 273

Chini, R., Elsaesser, H., \& Neckel, T. 1980, A\&A, 91, 186

Crété, E., Giard, M., Joblin, C., et al. 1999, A\&A, 352, 277

Croiset, B. A., Candian, A., Berné, O., \& Tielens, A. G. G. M. 2016, A\&A, 590, A26

Draine, B. T. 2003, ARA\&A, 41, 241

Draine, B. T., \& Li, A. 2007, ApJ, 657, 810

Egusa, F., Wada, T., Sakon, I., et al. 2013, ApJ, 778, 1

Fleming, B., France, K., Lupu, R. E., \& McCandliss, S. R. 2010, ApJ, 725, 159

Galliano, F., Madden, S. C., Tielens, A. G. G. M., Peeters, E., \& Jones, A. P. 2008, ApJ, 679, 310

Giard, M., Bernard, J. P., \& Dennefeld, M. 1992, A\&A, 264, 610

Giard, M., Bernard, J. P., Lacombe, F., Normand, P., \& Rouan, D. 1994, A\&A, 291, 239

Hanson, M. M., Howarth, I. D., \& Conti, P. S. 1997, ApJ, 489, 698

Haraguchi, K., Nagayama, T., Kurita, M., Kino, M., \& Sato, S. 2012, PASJ, 64, 127 
Hony, S., Van Kerckhoven, C., Peeters, E., et al. 2001, A\&A, 370, 1030

Houck, J. R., Roellig, T. L., van Cleve, J., et al. 2004, ApJS, 154, 18

Jiang, Z., Yao, Y., Yang, J., et al. 2002, ApJ, 577, 245

Joblin, C., Tielens, A. G. G. M., Geballe, T. R., \& Wooden, D. H. 1996, ApJ, 460, L119

Kaneda, H., Onaka, T., \& Sakon, I. 2005, ApJ, 632, L83

Kaneda, H., Suzuki, T., Onaka, T., Okada, Y., \& Sakon, I. 2008, PASJ, 60, S467

Kaneda, H., Ishihara, D., Kobata, K., et al. 2014, Planet. Space Sci., 100, 6

Kondo, T., Ishihara, D., Kaneda, H., et al. 2016, AJ, 151, 71

Lada, C. J., Depoy, D. L., Merrill, K. M., \& Gatley, I. 1991, ApJ, 374, 533

Lebouteiller, V., Barry, D. J., Spoon, H. W. W., et al. 2011, ApJS, 196, 8

Minamidani, T., Nishimura, A., Miyamoto, Y., et al. 2016a, in Millimeter, Submillimeter, and FarInfrared Detectors and Instrumentation for Astronomy VIII, Vol. 9914, 99141Z-99141Z-10

Minamidani, T., Umemoto, T., Nishimura, A., et al. 2016b, in EAS Publications Series, Vol. 75, EAS Publications Series, 193-194

Nagashima, C., Nagayama, T., Nakajima, Y., et al. 1999, in Star Formation 1999, ed. T. Nakamoto, $397-398$

Nagayama, T., Nagashima, C., Nakajima, Y., et al. 2003, in Society of Photo-Optical Instrumentation Engineers (SPIE) Conference Series, Vol. 4841, Instrument Design and Performance for Optical/Infrared Ground-based Telescopes, ed. M. Iye \& A. F. M. Moorwood, 459-464

Peeters, E., Hony, S., Van Kerckhoven, C., et al. 2002, A\&A, 390, 1089

Pérez-Beaupuits, J. P., Wiesemeyer, H., Ossenkopf, V., et al. 2012, A\&A, 542, L13

Pilleri, P., Montillaud, J., Berné, O., \& Joblin, C. 2012, A\&A, 542, A69

Povich, M. S., Stone, J. M., Churchwell, E., et al. 2007, ApJ, 660, 346

Rapacioli, M., Joblin, C., \& Boissel, P. 2005, A\&A, 429, 193

Reach, W. T., Abergel, A., Boulanger, F., et al. 1996, A\&A, 315, L381

Ricca, A., Bauschlicher, Jr., C. W., Boersma, C., Tielens, A. G. G. M., \& Allamandola, L. J. 2012, ApJ, 754, 75

Sakon, I., Onaka, T., Wada, T., et al. 2007, PASJ, 59, S483 
Sheffer, Y., \& Wolfire, M. G. 2013, ApJ, 774, L14

Skrutskie, M. F., Cutri, R. M., Stiening, R., et al. 2006, AJ, 131, 1163

Smith, J. D. T., Armus, L., Dale, D. A., et al. 2007a, PASP, 119, 1133

Smith, J. D. T., Draine, B. T., Dale, D. A., et al. 2007b, ApJ, 656, 770

Stock, D. J., Choi, W. D.-Y., Moya, L. G. V., et al. 2016, ApJ, 819, 65

Stutzki, J., \& Guesten, R. 1990, ApJ, 356, 513

Tielens, A. G. G. M. 2005, The Physics and Chemistry of the Interstellar Medium

-. 2008, ARA\&A, 46, 289

Xu, Y., Moscadelli, L., Reid, M. J., et al. 2011, ApJ, 733, 25

Yamagishi, M., Kaneda, H., Ishihara, D., et al. 2010, PASJ, 62, 1085

-. 2012, A\&A, 541, A10 


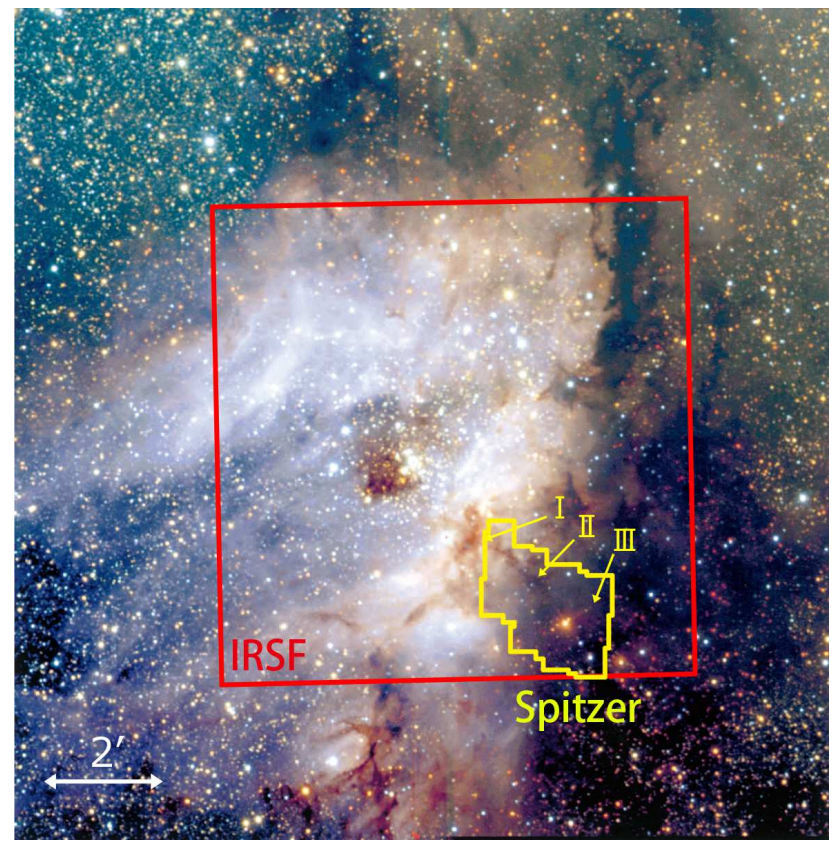

Fig. 1.- Regions observed with Spitzer and IRSF overlaid on the near-IR three color image of M17 (Jiang et al. 2002). I-III indicate the positions where the mid-IR spectra in Fig. 2 are extracted. 


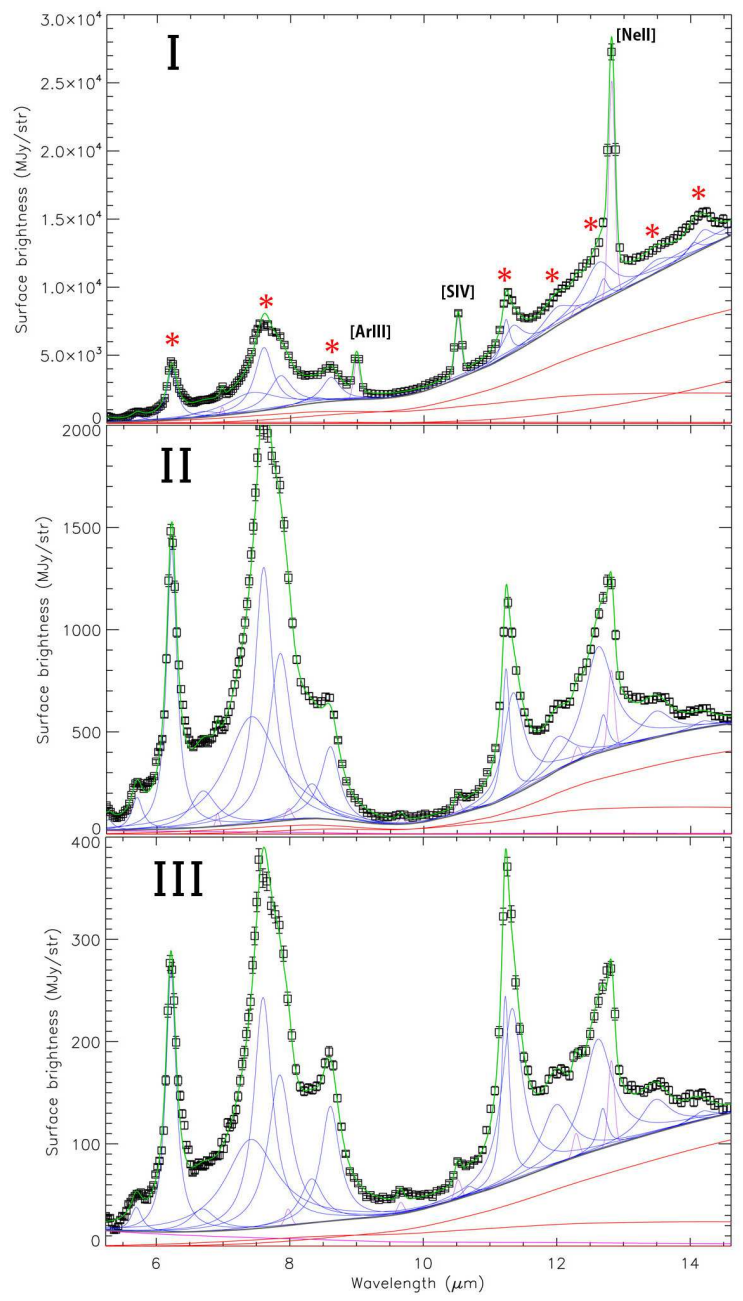

Fig. 2. - Examples of the mid-IR spectra extracted from the areas of $3^{\prime \prime} .6 \times 3^{\prime \prime} .6$, which are named as positions I-III in Fig. 1. Red asterisks indicate PAH features at wavelengths of 6.2, 7.7, 8.6, $11.3,12.0,12.7,13.5$, and $14.2 \mu \mathrm{m}$. The green curve indicates the best-fit model using PAHFIT (Smith et al. 2007b), while the blue, magenta, and red curves indicate the best-fit components of the PAH features, fine-structure lines, and continuum emission, respectively. 


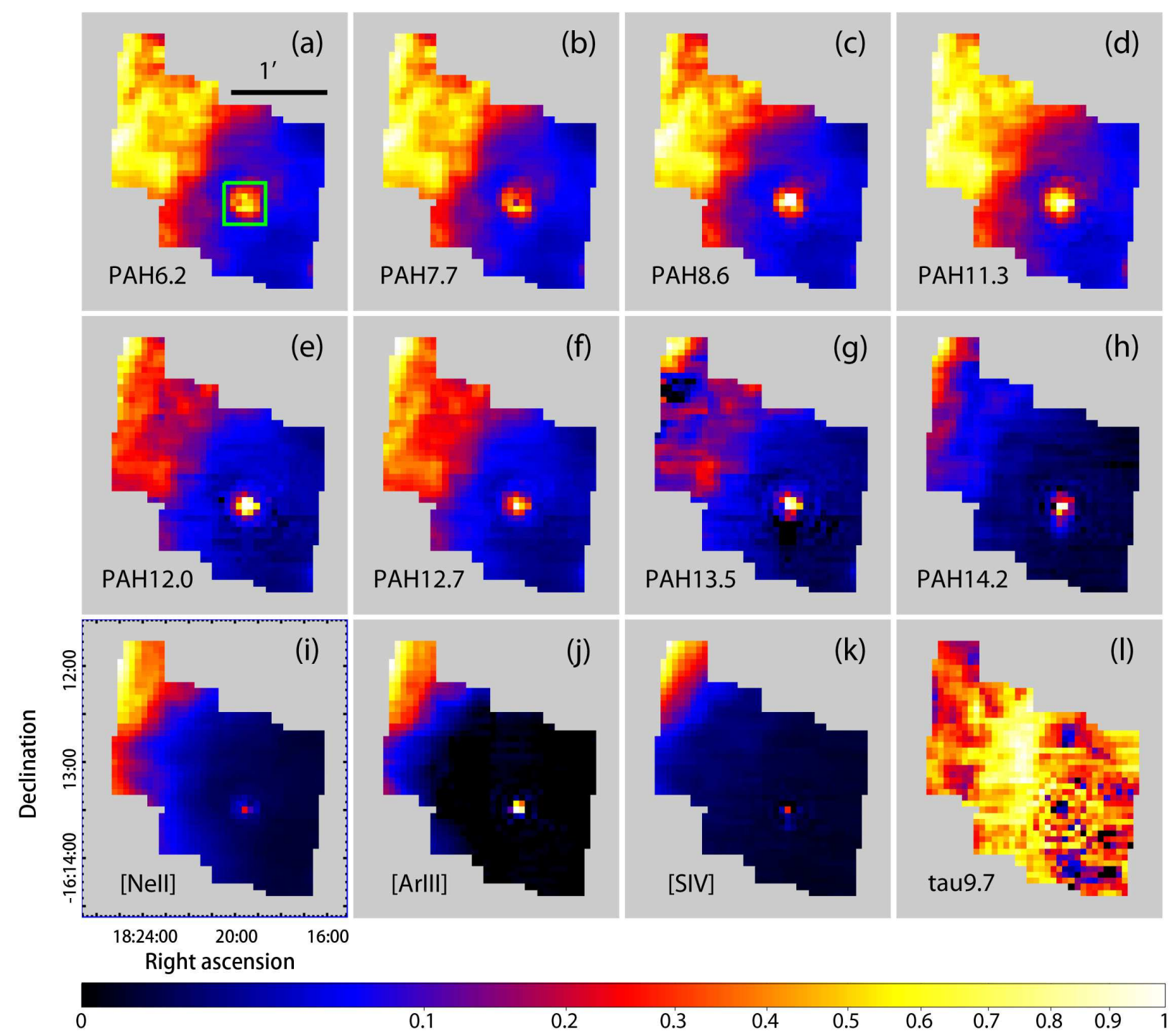

Fig. 3.- Spectral maps of the (a) PAH $6.2 \mu \mathrm{m}$, (b) PAH $7.7 \mu \mathrm{m}$, (c) PAH $8.6 \mu \mathrm{m}$, (d) PAH $11.3 \mu \mathrm{m}$, (e) PAH $12.0 \mu \mathrm{m}$, (f) PAH $12.7 \mu \mathrm{m}$, (g) PAH $13.5 \mu \mathrm{m}$, (h) PAH $14.2 \mu \mathrm{m}$ features, the (i) $[\mathrm{NeII}],(\mathrm{j})[\mathrm{ArIII}]$, and (k) [SIV] lines, and (l) optical depth of silicate dust absorption at $9.7 \mu \mathrm{m}$ obtained with PAHFIT (Smith et al. 2007b). The maps are normalized by the following maximum values: (a) $1.2 \times 10^{-4} \mathrm{~W} / \mathrm{m}^{2} / \mathrm{sr}$, (b) $3.8 \times 10^{-4} \mathrm{~W} / \mathrm{m}^{2} / \mathrm{sr}$, (c) $6.2 \times 10^{-5} \mathrm{~W} / \mathrm{m}^{2} / \mathrm{sr}$, (d) $6.0 \times 10^{-5} \mathrm{~W} / \mathrm{m}^{2} / \mathrm{sr}$, (e) $3.7 \times 10^{-5} \mathrm{~W} / \mathrm{m}^{2} / \mathrm{sr}$, (f) $7.2 \times 10^{-5} \mathrm{~W} / \mathrm{m}^{2} / \mathrm{sr}$, (g) $2.6 \times 10^{-5} \mathrm{~W} / \mathrm{m}^{2} / \mathrm{sr}$, (h) $2.6 \times 10^{-5} \mathrm{~W} / \mathrm{m}^{2} / \mathrm{sr}$, (i) $4.5 \times 10^{-5} \mathrm{~W} / \mathrm{m}^{2} / \mathrm{sr}$, (j) $2.3 \times 10^{-5} \mathrm{~W} / \mathrm{m}^{2} / \mathrm{sr}$, (k) $3.6 \times 10^{-5} \mathrm{~W} / \mathrm{m}^{2} / \mathrm{sr}$, and (l) 1.5. The area indicated by the green box in panel (a) includes a well known young stellar object, where the spectra show ice absorption features and are not fitted well with PAHFIT. 


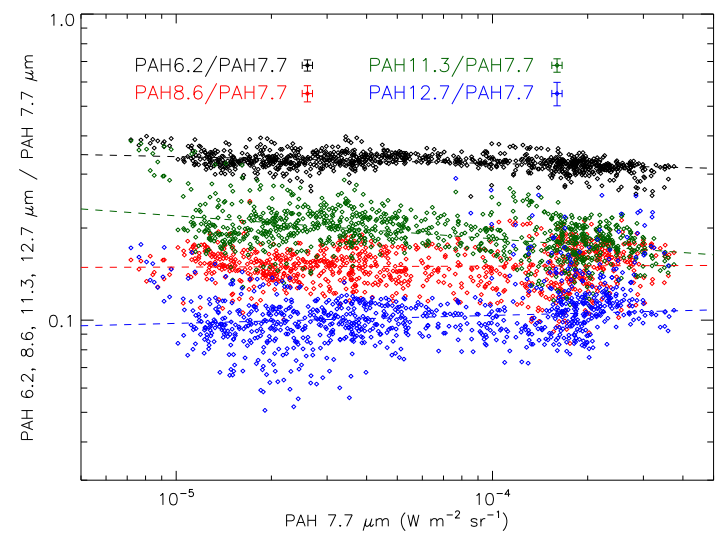

Fig. 4.- Interband ratios of the PAH $6.2 \mu \mathrm{m}, 8.6 \mu \mathrm{m}, 11.3 \mu \mathrm{m}$, and $12.7 \mu \mathrm{m}$ features to the PAH $7.7 \mu \mathrm{m}$ feature plotted against intensities of the PAH $7.7 \mu \mathrm{m}$ feature. The median value of the uncertainties for each interband ratio is shown as a typical error bar in the figure legend. The dashed lines indicate the best-fit power-law relation for each interband ratio.
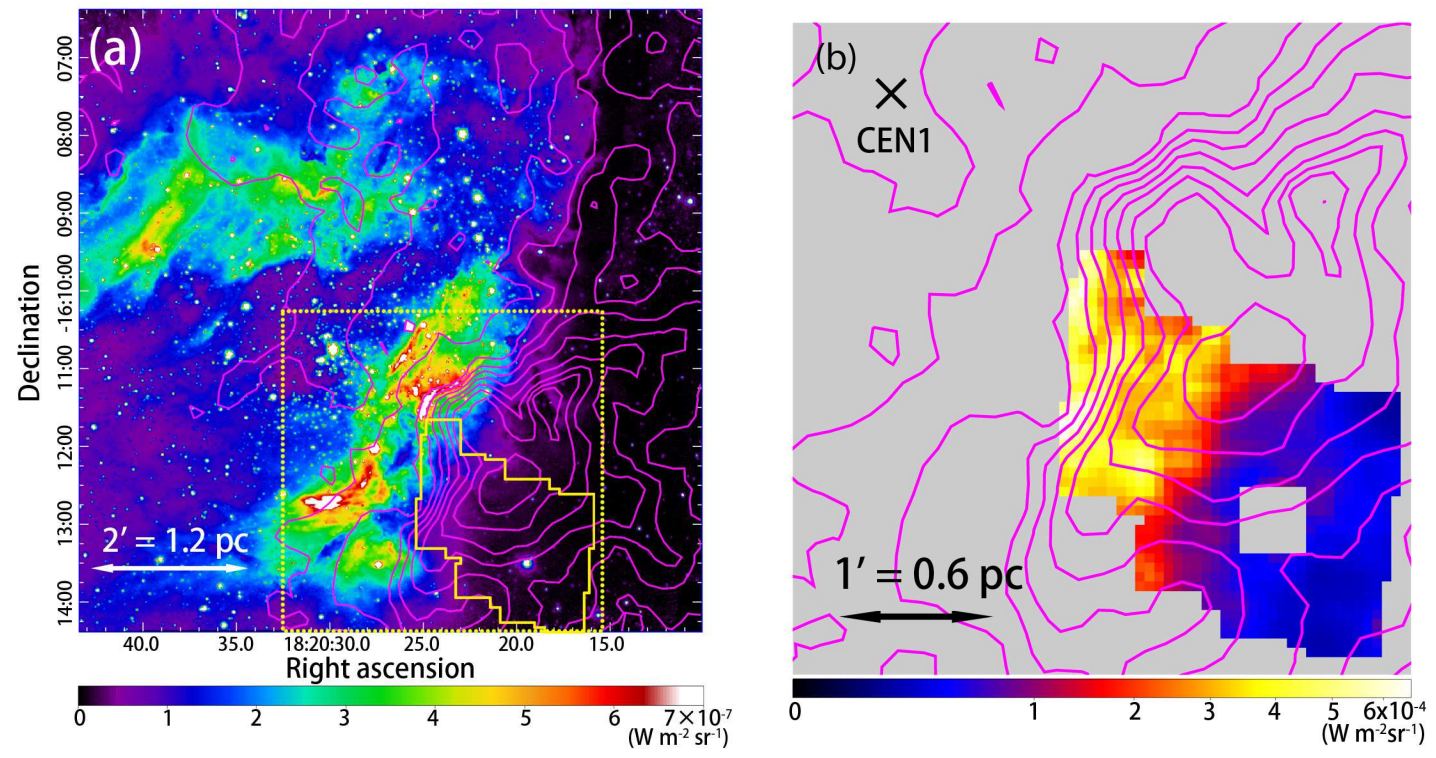

Fig. 5.- (a) $\mathrm{Br} \gamma$ narrow-band image of M17 taken with IRSF, where the contours are the ${ }^{13} \mathrm{CO}$ $(J=1-0)$ intensity integrated over the velocity range of $10-30 \mathrm{~km} / \mathrm{s}$. The contours are drawn at ten linearly spaced levels from the $5 \sigma$ detection level of $20 \mathrm{~K} \mathrm{~km} / \mathrm{s}$ to the peak of $206 \mathrm{~K} \mathrm{~km} / \mathrm{s}$. The yellow box indicates the observed region with Spitzer/IRS. The area indicated by the yellow dotted box is enlarged in panel (b). (b) Total intensity map of the major PAH features (PAH $6.2 \mu \mathrm{m}$, PAH $7.7 \mu \mathrm{m}$, PAH $8.6 \mu \mathrm{m}$, PAH $11.3 \mu \mathrm{m}$, and PAH $12.7 \mu \mathrm{m})$ overlaid on the ${ }^{13} \mathrm{CO}(J=1-0)$ contour map. The cross represents the position of the most prominent O4-type star in M17, CEN1. The region indicated by the green box in Fig. 3(a) is masked. 

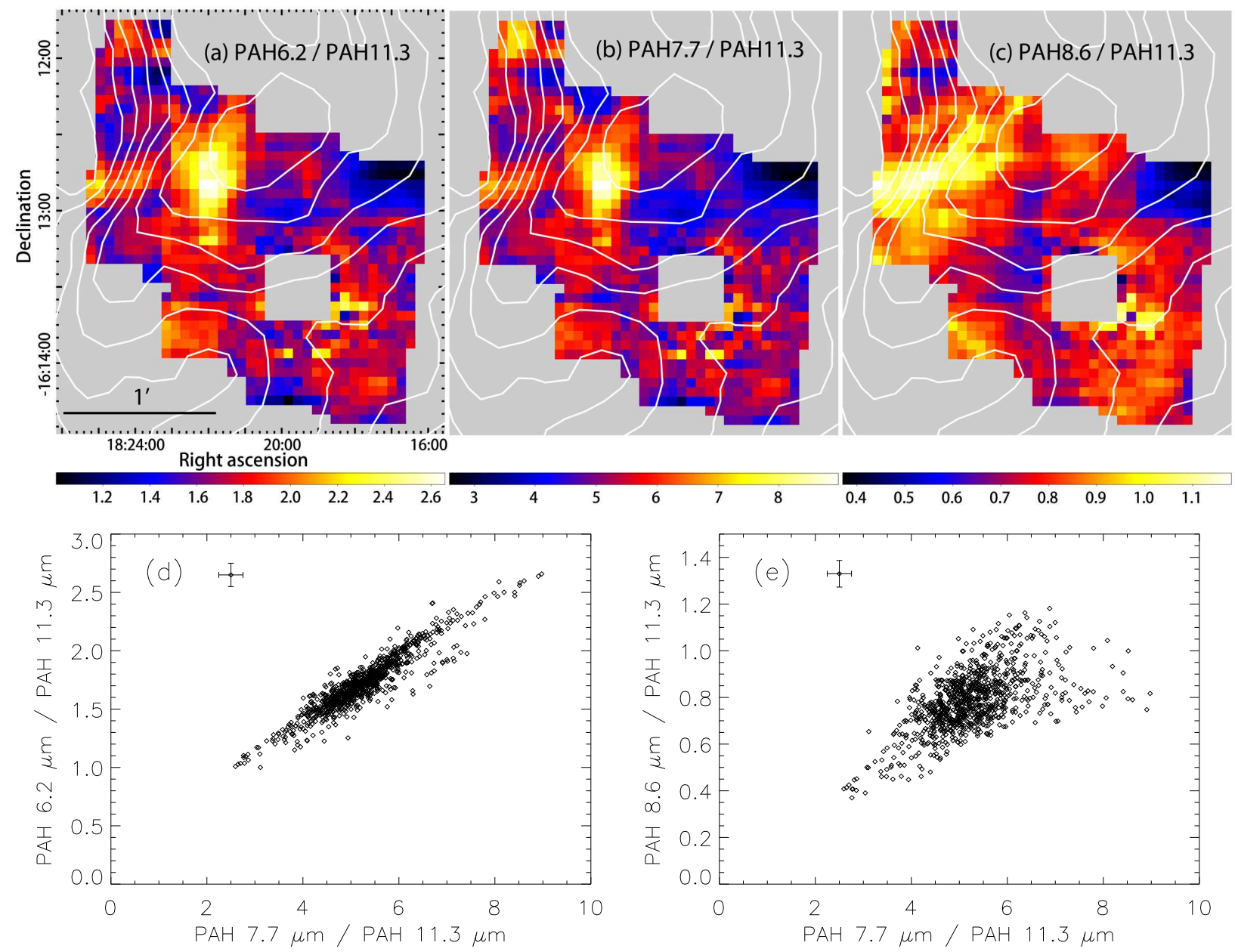

Fig. 6.- (a-c) Interband ratio maps of the PAH $6.2 \mu \mathrm{m}, 7.7 \mu \mathrm{m}$, and $8.6 \mu \mathrm{m}$ features to the PAH $11.3 \mu \mathrm{m}$ feature. The contours are the same as shown in Fig. 5, The region indicated by the green box in Fig. 3(a) is masked. (d, e) Correlation plots between the above interband ratios. A typical error is shown on the upper left corner. 

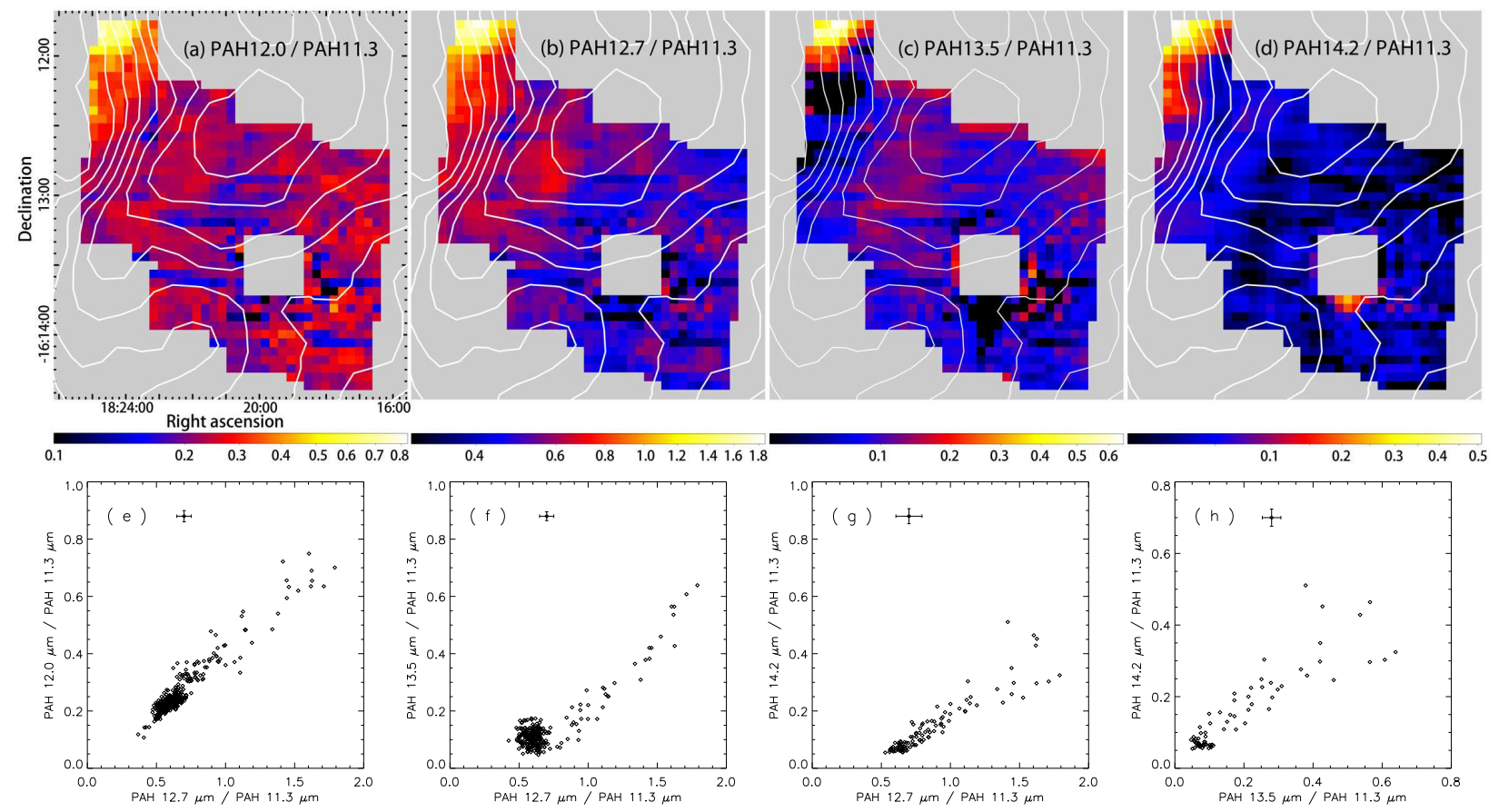

Fig. 7.- (a-d) Interband ratio maps of the PAH $12.0 \mu \mathrm{m}, 12.7 \mu \mathrm{m}, 13.5 \mu \mathrm{m}$, and $14.2 \mu \mathrm{m}$ features to the PAH $11.3 \mu \mathrm{m}$ feature. The contours are the same as shown in Fig. 5 . The region indicated by the green box in Fig. 3(a) is masked. (e-h) Examples of correlation plots between the above interband ratios. The interband ratios are plotted for regions showing strong PAH emissions (more than $10 \%$ of the peak intensity in Fig. 3). A typical error is shown on the upper left. 


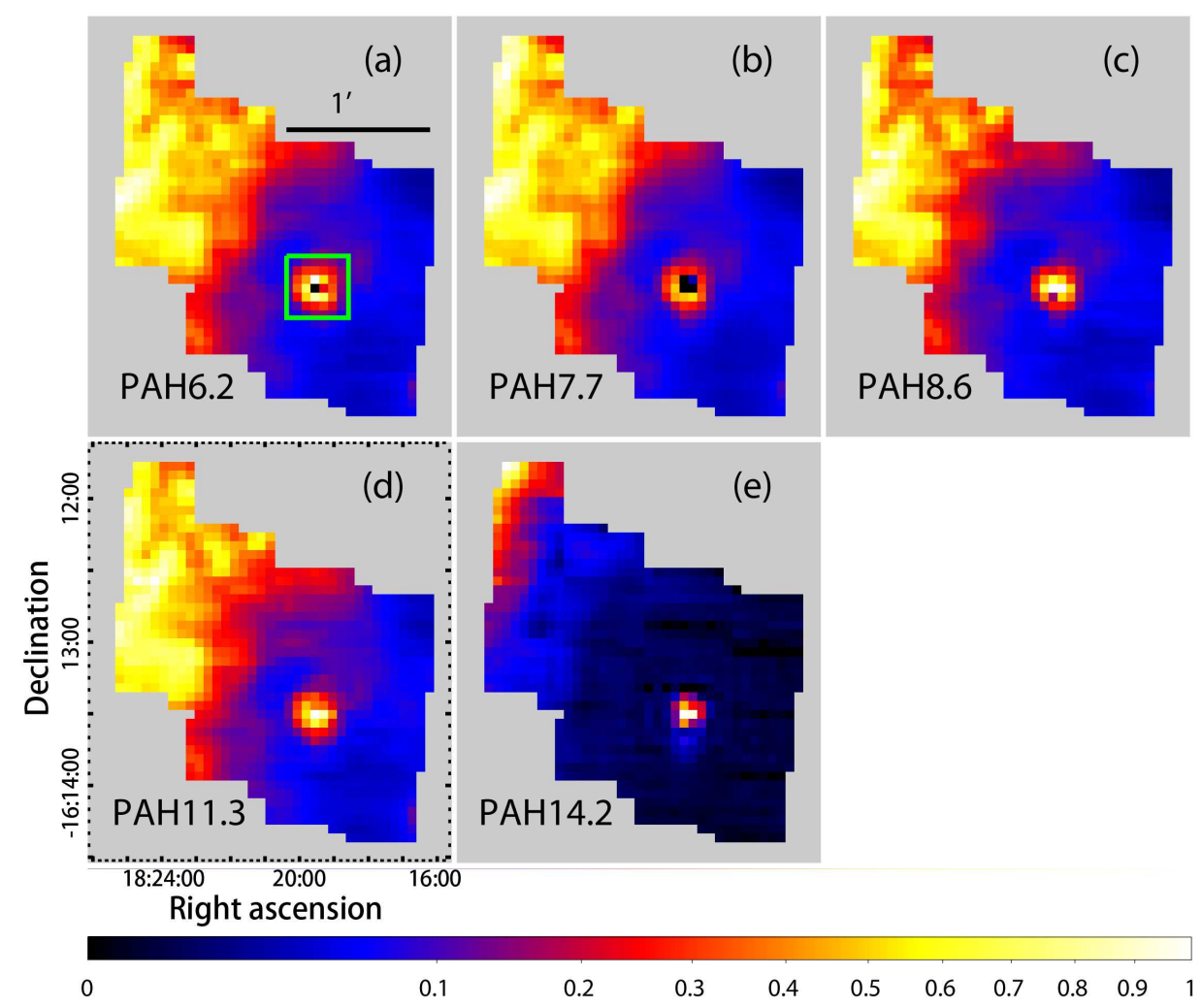

Fig. 8.- Spectral maps of the (a) PAH $6.2 \mu \mathrm{m}$, (b) PAH $7.7 \mu \mathrm{m}$, (c) PAH $8.6 \mu \mathrm{m}$, (d) PAH $11.3 \mu \mathrm{m}$, and (e) PAH $14.2 \mu \mathrm{m}$ features obtained with the linear-baseline-fitting method. The maps are normalized by the following maximum values: (a) $6.5 \times 10^{-5} \mathrm{~W} / \mathrm{m}^{2} / \mathrm{sr}$, (b) $1.3 \times 10^{-4} \mathrm{~W} / \mathrm{m}^{2} / \mathrm{sr}$, (c) $1.4 \times 10^{-5} \mathrm{~W} / \mathrm{m}^{2} / \mathrm{sr}$, (d) $3.0 \times 10^{-5} \mathrm{~W} / \mathrm{m}^{2} / \mathrm{sr}$, and (e) $1.5 \times 10^{-5} \mathrm{~W} / \mathrm{m}^{2} / \mathrm{sr}$. The area indicated by the green box in Fig. 3(a) is also shown in panel (a).

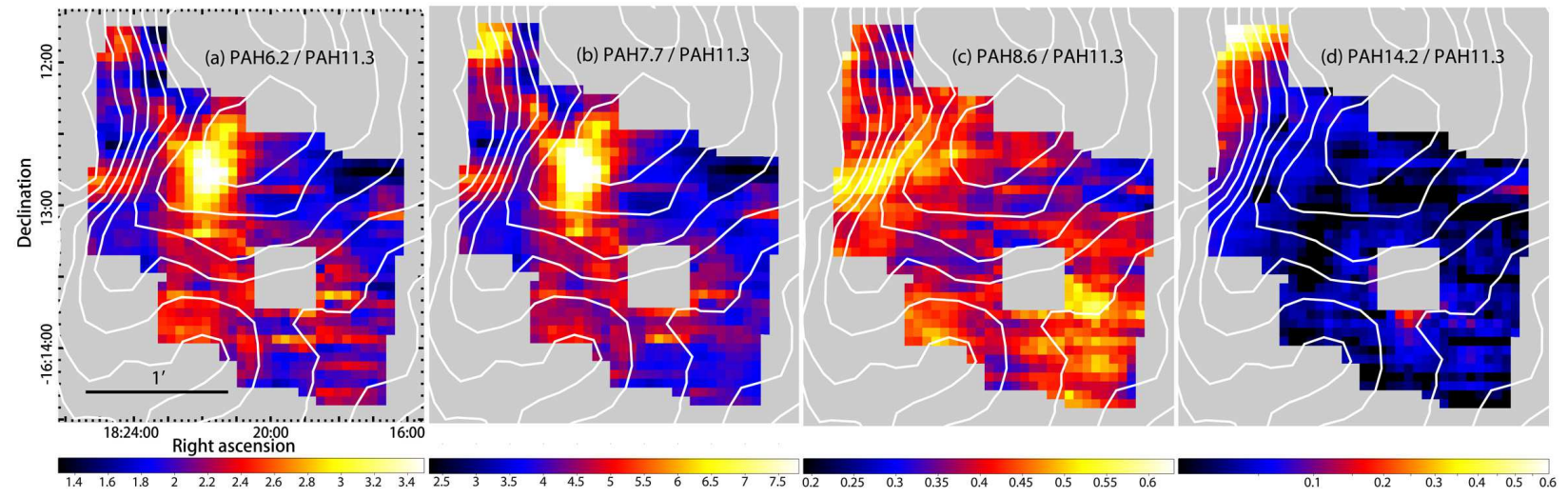

Fig. 9.- Examples of the PAH interband ratio maps derived with the linear-baseline-fitting method. The region indicated by the green box in Fig. 3(a) is masked. The contours are the same as shown in Fig. 5 , 


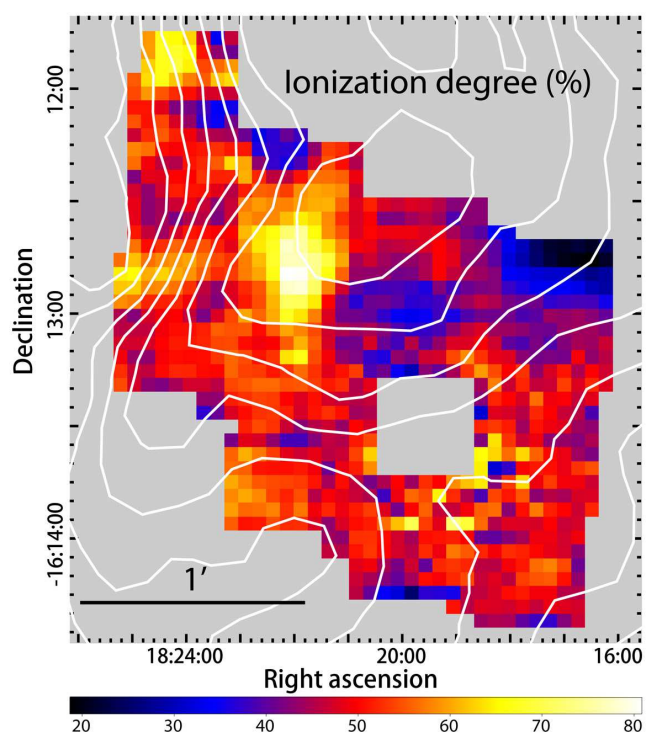

Fig. 10. - Degree of PAH ionization estimated from the PAH $7.7 \mu \mathrm{m} / \mathrm{PAH} 11.3 \mu \mathrm{m}$ ratios. The contours are the same as shown in Fig. 5. The region indicated by the green box in Fig. 3(a) is masked.

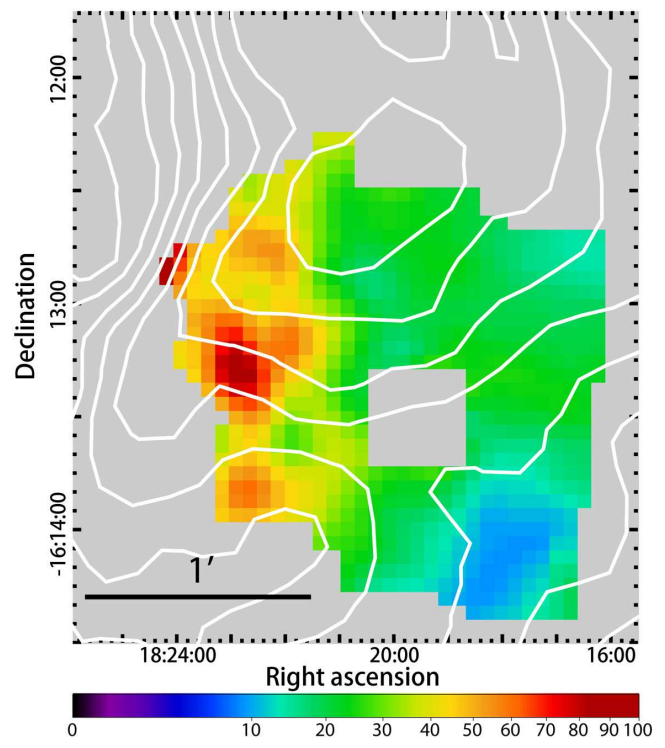

Fig. 11. - Distribution of $G_{0}$ estimated from the Herschel/PACS 70 and $100 \mu \mathrm{m}$ maps. The color bar is shown in the square root scale. The spatial resolution of the map is $6 .^{\prime \prime} 7$ which is the PSF size of the PACS $100 \mu \mathrm{m}$ map. The contours are the same as shown in Fig. 5. The regions showing strong $[\mathrm{NeII}]\left(>5.0 \times 10^{-6} \mathrm{~W} / \mathrm{m}^{2} / \mathrm{sr}\right)$ are masked. 


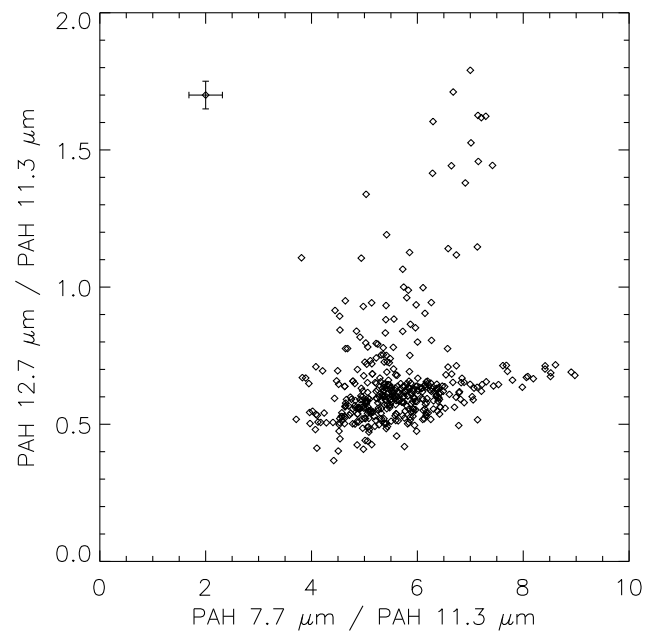

Fig. 12.- PAH $12.7 \mu \mathrm{m} / \mathrm{PAH} 11.3 \mu \mathrm{m}$ ratios versus the PAH $7.7 \mu \mathrm{m} / \mathrm{PAH} 11.3 \mu \mathrm{m}$ ratios plotted for regions showing strong PAH emissions (more than $10 \%$ of the peak intensity in Fig. 3). A typical error is shown on the upper left. 